\title{
Investigación conceptual sobre turismo sexual
}

\author{
Conceptual research on sex tourism
}

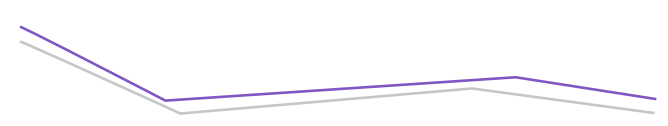

Celeste Nava-Jiménez*凶, Andrea Robles-Juárez**, Brandon Iván RoqueRodríguez***, Bianca Marysol Vargas-Fonseca*****

Nava-Jiménez, C., Robles-Juárez, A., Roque-Rodríguez, B. I., \& Vargas-Fonseca, B. M. (2018). Investigación conceptual sobre turismo sexual. Investigación y Ciencia de la Universidad Autónoma de Aguascalientes, 26(75), 73-80.

\section{RESUMEN}

El turismo sexual es un fenómeno con importantes implicaciones en el ámbito social, territorial y económico. Es un tema controversial en la literatura turística y es poco común que sea abordado desde la investigación conceptual. Este tipo de investigación ha tenido avances, pero aún no tiene la suficiente representatividad en los estudios turísticos, lo que causa que incluso definir el concepto sea un tanto complicado y con opiniones dispersas. En la presente investigación se ha empleado como el tipo de investigación más adecuada para poder abordar el turismo sexual como objeto de estudio conceptual en los estudios turísticos, para conocer en primer instancia las dimensiones teóricas del concepto, seguido de la posibilidad de romper paradigmas teóricos y sociales que puede provocar el concepto

Palabras clave: investigación; destino; turismo; turismo sexual; turista; sociedad.

Keywords: destination; research; tourism; sex tourism; society; tourist.

\section{Recibido: 2 de enero de 2018, aceptado: 14 de junio de 2018}

* Facultad de Turismo y Gastronomía, Universidad De La Salle Bajío. Av. Universidad 602, Col. Campestre, C. P. 37150, León, Guanajuato, México. Correo electrónico: celenava@gmail.com; cnava@delasalle.edu. $\mathrm{mx}$

** Departamento de Turismo, Recreación y Servicio, Universidad de Guadalajara. Calle Parián 1894 interior J-7, Colonia Jardines del Country, C. P. 44210, Guadalajara, Jalisco, México. Correo electrónico: andrea. rj.tur@gmail.com

*** Escuela Superior de Turismo, Instituto Politécnico Nacional. Héctor Espinoza no 535, Colonia Escuadrón 201, C. P. 09060, Delegación Iztapalapa, D. F., México. Correo electrónico: brandon.roque@outlook.es

**** Unidad Académica de Turismo, Universidad Autónoma de Nayarit. Bahía de Guaymas no. 14, Colonia Centro La Peñita de Jaltemba, C. P. 63726, Nayarit, México. Correo electrónico: marysol.vf9@gmail.com

$凶$ Autor para correspondencia de turismo sexual. Finalmente, el trabajo reflexiona para comprender mejor las implicaciones y conceptos que giran alrededor de él y que han colaborado para poder conceptualizarlo y analizarlo.

\section{ABSTRACT}

The phenomenon of sex tourism has important implications on social, territorial and economic environments. It is a controversial matter in the tourism literature and is rare to be addressed through conceptual research. This type of research does not have enough representation in tourism studies despite the advances found, so it is difficult to define the concept due the different opinions. In this article, conceptual research has been chosen as the most suitable type of research to address sex tourism as an object of conceptual study, in order to know at first the theoretical dimensions of the concept, following the possibility of breaking some theoretical and social paradigms about sex tourism. Finally, the study reflect on the implications and concepts that revolve around it and will help to define it, conceptualize and analyze it.

\section{INTRODUCCIÓN}

Este estudio se concretó durante el Programa Interinstitucional para el Fortalecimiento de la Investigación y el Posgrado del Pacífico Verano Delfín 2016, en la Universidad de Guanajuato, mediante una lectura crítica y conceptual sobre el turismo sexual como categoría de análisis, de la revista Estudios y Perspectivas en Turismo, en su volumen 24 de 2015 y de algunos documentos adicionales en inglés. Para efectuar la revisión se 
IIVESTIGACIÓn Y CIERCIA DE LA UNIVERSIDAD AUTÓNOMA

establecieron palabras clave en español y en inglés y se utilizó un software especializado en análisis de textos para una mejor comprensión de los mismos.

Es conocido que la investigación turística ha sido etiquetada entre lo cuantitativo, lo cualitativo y lo mixto; sin embargo, esto no quiere decir que la investigación conceptual en turismo no existe. De acuerdo con Xin, Tribe y Chambers (2013) es el tipo de investigación donde se puede progresar sin información empírica o inmediata, sin datos estadísticos que sustenten la creación del conocimiento. Por tanto, la investigación conceptual sobre el turismo sexual, objeto de estudio de esta investigación, se hace a partir de un análisis literario que gira alrededor de este concepto. El hilo conductor de la presente gira en torno a la crítica y revisión del concepto turismo sexual en la literatura turística, por ello es importante revisar la interpretación de este concepto en el material existente y hacer una revisión conceptual para que en la posteridad se puedan seguir concatenando esfuerzos teóricos y empíricos sobre este tema. Según Gray, Williamson y Karp (2007) y Kothari (2008) citados en Xin, Tribe y Chambers (2013), la posibilidad de creatividad e innovación se potencializa en la investigación conceptual. Una característica más, citando a Leuzinger-Bohleber y Fischmann (2006) en Xin et al. (2013), es que la investigación conceptual ayuda a construir nuevos puentes entre disciplinas cercanas.

La investigación conceptual en el tema estudiado implica el rompimiento de paradigmas que la tradición científica ha dejado como herencia en la construcción del conocimiento turístico y que, como ya es conocido, se limita a lo cuantitativo y a lo cualitativo. En este sentido, el turismo sexual debe ser comprendido desde su esencia y significado. Desde esta perspectiva, el estudio del turismo sexual como concepto podrá crear puentes entre lo que es turístico y la conducta del visitante en el destino, desde los puntos de vista sociológico y antropológico.

Según Xin et al. (2013, pp. 77-78), "la investigación conceptual en turismo está en aumento; en términos cuantitativos y cualitativos ha tenido avances, pero aun así la investigación conceptual en turismo, según los autores, no tiene representatividad". Uno de los objetivos de esta investigación es aproximarse a una comprensión de la conceptualización que de turismo sexual se tiene a partir de la revisión de bibliografía que permite un acercamiento teórico-conceptual al objeto de estudio, en este caso turismo sexual como concepto. De este modo, uno de los tres resultados importantes de la investigación conceptual según Xin et al. $(2013$, p. 66) es que

se genera una nueva definición del concepto estudiado, los otros resultados son: la investigación conceptual, mientras aumenta su popularidad, parece ser relativamente marginal en turismo, pero no asi en otras disciplinas o áreas, y la posible construcción de una tipología de los temas de la investigación conceptual.

Con respecto al turismo sexual y sus antecedentes, según Sánchez Mendoza y Santamaría Gómez (2015), el imaginario del turismo de sol y playa propone la idea de que a través de él se obtiene el verdadero placer de la aventura sexual, una idea a veces abierta y en ocasiones subliminal. En este sentido Azaola (2006) y López y Van Broeck (2013) comentan que México sobresale entre los países latinoamericanos en los que el turismo sexual se ha desarrollado, sobre todo en destinos turísticos de playa como Acapulco, Cancún y Puerto Vallarta, así como en ciudades fronterizas como Tijuana y Ciudad Juárez (citado en Bringas \& Gaxiola, 2015, p. 826).

Bringas y Gaxiola (2015, p. 826) rescatan la idea de Carter (2000), así como la de Baver y McKercher (2003) para asegurar que la industria del sexo en el destino es también utilizada por los residentes locales y que la idea de que solo los turistas participan en relaciones sexuales comerciales en el destino es gracias al turismo que promueve la prostitución. Aunque la prostitución no lo es todo, Oppermann (1999) y Hall (1994) mencionan que esta no puede ser vista como el único elemento que da fuerza y forma al turismo sexual, que limitar el turismo sexual únicamente a la misma es una forma demasiado simple de entender un fenómeno mucho más grande, que esto solo representa una pequeña porción de todo lo que puede estar involucrado dentro del turismo sexual (citados por Carr, 2016). Asimismo, Hall menciona que considerar siempre al proveedor del servicio sexual como prostituta o prostituto, no siempre es correcto o no siempre sucede así.

Investigar conceptualmente al turismo sexual da la posibilidad de relacionar ideas de formas nunca antes pensadas, como otra de las características de la investigación conceptual (Xin et al., 2013), de esta manera se puede crear conocimiento nuevo y crítico con nuevas relaciones e implicaciones. 
El método de trabajo fue la revisión documental del volumen 24 de la revista Estudios y Perspectivas en Turismo del Centro de Investigaciones y Estudios Turísticos de Argentina, los artículos versan sobre turismo y sexo. La lectura de los mismos, así como otros documentos que se consideraron de interés para el tema, se colocaron bajo el software Mendeley, por medio del cual se buscaron palabras claves como sexo, turismo, turista, sociedad, gay, turismo sexual, entre otras, con la finalidad de conocer la relación de los conceptos con el texto. De esta manera se pudo contrastar la visión de estos conceptos en los artículos revisados para poder construir y discutir el tema. Los resultados de la revisión teórica conducen a construir los apartados del artículo para discutir sobre las distintas visiones del tema de estudio que se reflejan en los documentos revisados.

\section{El anonimato del turismo sexual}

Según Veijola y Jokinen (1994) citados en Mendoza (2015, p. 890) del concepto de turismo sólo se ha contextualizado la parte positiva; es decir, aquella que está orientada al descanso y la recreación; hasta cierto punto la erudición del turismo sigue dominado por el conocimiento occidental y la tradición de la investigación científica (Huang, Van der Veen, \& Zhang, 2014). Por tanto, las variables de investigación responden a la necesidad de saber la cantidad de visitantes que llegan, hábitos de consumo, derrama económica, entre otras. En este sentido, el tema del sexo o turismo sexual no es tan considerado en los análisis e investigaciones turísticas, recientemente McKercher (2016) lo integra como un tipo de producto turístico que responde a una familia de productos turísticos indulgent $o$ indulgente-condescendiente que responden a la necesidad de placer en su taxonomía propuesta para productos turísticos.

Es muy pobre el conocimiento y comprensión de un asunto tan complejo y disperso (Carr, 2016), a pesar de que existe un progreso con respecto a las investigaciones en el área turística; aún es evidente la renuencia a tomar el tema de turismo sexual, analizarlo y presentarlo en su realidad, como bien lo mencionaron Hall (1994) y Graburn (1983) citados por Carr (2016). El concepto de turismo sexual se ha tomado como objeto de estudio por Clift y Forrest (1999), Hughes, Monterrubio y Miller (2010) y López y Van Broeck (2013), se puede decir que el concepto no ha sido estudiado por mucho tiempo, se considera relativamente nuevo en la literatura turística, mientras que su práctica ha sido desde tiempo atrás. Mendoza (2015) establece que "el concepto se consideraba un tema tabú en las décadas anteriores y por lo cual, no era un tema de estudio" (p. 890).

Según Blackburn, Tayor y Davis (2011) citados por Carr (2016), el turismo sexual se define como el acto de viajar donde la principal motivación del turista es conseguir sexo. Si las condiciones lo permiten el turista podría crear una identidad no propia de sí misma que le permita tener acceso a ciertos servicios, mientras que en el lugar de origen no se podría, ya que en un momento determinado se puede demostrar la falsedad de la persona.

En este orden de ideas, Prat (2010) en Monterrubio (2015, p. 909) cree que "el turismo es el que ha desenfrenado prácticas inmorales, ya que las personas se animan a probar cosas nuevas o a hacer lo que no harían en su lugar de residencia". Incluso podría hacerse referencia en cuestión de prácticas inmorales a lo que expresa Vargas \& Alcalá (2015, p. 870) citando a Díaz (2002) en su artículo sobre todo lo referente a la prostitución infantil en Acapulco desde el costo, los servicios que se ofertan hasta las investigaciones por parte del Sistema Nacional para el Desarrollo Integral de la Familia en México (SNDIF - DIF) señalando que las autoridades participan en conjunto para que se explote sexualmente a los niños. Es importante recalcar que si se mira de manera más profunda y fría el fenómeno en estudio, toparse con la presencia del turismo sexual infantil representa la forma más atroz del fenómeno (Frohlick, 2010, citado en Carr, 2016).

Por su parte, Córdova Plaza (2015, p. 849) menciona que la prostitución tiene diferentes puntos de análisis y eso se contradice en las sociedades modernas, ya que es considerada ilegal pero las autoridades conocen los lugares donde ocurre y gran parte de las veces obtienen algún beneficio monetario de la misma, lo que implica colusión en actividades que dañan a la sociedad local y turística. El caso más próximo es el Hotel Gran Vía en la ciudad de Toluca, Estado de México, donde existía una red de prostitución para facilitar el turismo sexual en la zona, como se encuentra en la nota de Excélsior (Redacción, 26 de noviembre de 2017) y en la de Regeneración (DDD, 25 de noviembre de 2017).

"La búsque da de la satisfacción sexual es hoy día más abierta y frecuente", así lo confirman Sánchez 
IIVESTIGACIÓn Y CIERCIA DE LA UNIVERSIDAD AUTÓNOMA
Mendoza y Santamaría Gómez (2015, p. 1004) cuando leen a lan Littlewood (2002), el cual sostiene que "la motivación sexual ha estado presente en el deseo de los turistas desde hace por lo menos un siglo en la sociedad digitalizada e hipermoderna". Tal es la presencia del sexo en turismo que la palabra sex o sexo ha sido incluida en las cuatro s de la actividad turística de sol y playa (Crick, 2001) por representar un grado de importancia para la actividad turística y para la promoción de esta. En efecto, los destinos de sol y playa, donde el sun, sand, sea y sex se envuelven en un coqueteo que permite la práctica del turismo sexual, en el que la gente que trabaja en bares, antros y estos sitios de encuentro, al igual que la comunidad local, saben distinguir a quienes prestan este tipo de servicios.

No está de más referirse a Carr (2016) cuando menciona que comúnmente "los destinos de sol y playa son fácilmente relacionados con cuestiones sexuales", simplemente al presentar en gran parte de su publicidad cuerpos semidesnudos, pero es importante aclarar que no siempre tiene que ser así. En un mundo tan sexualizado como el actual "el turismo no es más que otro producto o servicio que también ha sido alcanzado por estas insinuaciones", con lo que concuerdan Bishop y Robinson (1998) citados por Carr (2016). No obstante, "en una sociedad desarrollada del siglo XXI aún existe cierto tabú sobre los temas sexuales que conlleven ciertos gustos, preferencias o prácticas que no sean las esperadas por la sociedad", como expresa Carr (2016), el sexo tiene un encargo, el de ser escondido a los ojos del inofensivo. Frohlick (2010) citado por Carr (2016) acierta al expresar que "el sexo logra impactar casi en todas las facetas del turismo", por lo que es una actividad que no puede ser ignorada ni deslindada del mismo, sino que debe ser atendida seriamente para comprenderla mejor.

Si se considera el gran impacto que los nuevos sistemas de comunicación han tenido en las relaciones sociales es importante destacar cómo la sociedad contemporánea ha tecnificado las prácticas, llegando a modificar el uso y aprovechamiento del tiempo libre a través de las nuevas tecnologías, incluyendo y creando nuevas maneras de gestionar el tiempo libre. Desde esta perspectiva, según Hall (1992) citado por Carr (2016), el turismo sexual es un asunto que dentro de una dinámica internacional y globalizada, ha logrado ser propenso a sensacionalismo.
En esta sociedad digitalizada, uno de los mecanismos que se utiliza para aprovechar el tiempo libre es el internet que fomenta el intercambio intercultural y la movilidad que se han masificado hoy día (Córdova Plaza, 2015); en materia de turismo sexual, este se ha fomentado a partir de los encuentros de compañeros sexuales en las salas de chat, los sitios de encuentro y las aplicaciones que existen en la red, especialmente en los países de África, Asia y América Latina, como describen Piscitelli (2005) y McLean (2008), citados en Lanzarini (2015).

En la sociedad moderna, compleja y tecnificada, el capital es un factor importante para el desarrollo de la sociedad y, por supuesto, de los destinos turísticos, en este sentido el dinero puede ser elemento diferenciador entre turismo sexual y turismo erótico. A decir de Norrild (2007) citado en Arroyo y Amador (2015, p. 983), existe una distinción entre ambos, "el primero implicando un intercambio monetario y el segundo, así como el turismo romántico donde el sexo es voluntario".

Así como el capital, los negocios influyen en la dinámica económica del destino y del motivo principal del viaje y en este sentido Cáceres, Nureña y Gomero (2015) distinguen entre turismo sexual y el uso de servicios sexuales por parte de turistas y viajeros de negocios. En el primero, el visitante viaja con el propósito principal de mantener relaciones sexuales en el destino de su elección, mientras que en el segundo caso el turista cuya motivación de viaje es diferente puede o no hacer uso de los servicios sexuales en el destino. Esto implica que aunque exista un motivo de viaje específico no es garantía de que el turista cultural, el turista de negocios o cualquier otra categoría pueda hacer uso de servicios sexuales en el sitio visitado.

Carr (2016) hace referencia a Matter (2005), quien propone una definición un tanto diferente para los turistas sexuales, estableciendo diferencias entre los preferenciales, quienes viajan explícitamente con el propósito de tener relaciones, y los situacionales, quienes no planearon verse involucrados sexualmente con personas locales, pero aprovecharon la oportunidad cuando se presentó (citado en Samarasinghe, 2008). En este sentido existe una ruptura de la profesión que se tiene en el lugar de residencia (Lagunas, 2010); es decir, el turista puede "olvidar" su vida y su ocupación 


\section{IIVESTIGAGIÓn Y CUERCIA DE LA UחIVERSIDAD AUTÓNOMA DE RGUASCHLIETTES}

durante la existencia del viaje, lo que puede llevarlo a actuar de manera distinta.

Como bien mencionan McKercher y Baver (2003) citados por Carr (2016), el sexo es una parte natural de la vida, por tanto, no debería ser tan sorprendente que también se vea involucrado con el turismo. Y en este sentido Kibicho (2009) citado por Carr (2016), menciona que si las actividades sexuales son parte de las actividades normales que se realizan estando en el lugar de residencia habitual, es entendible que se busque realizar estas mismas actividades mientras se está de visita en otro lugar. Cuando se habla de hombres y mujeres en el turismo sexual existe una cierta inclinación según Carr (2016) para limitar que la categoría turismo sexual sea exclusiva de los hombres, mientras que para las mujeres debe conceptualizarse como un turismo de romance. Lo que lleva a Carr (2016) a cuestionar: ¿Por qué esto no puede verse al contrario, mujeres que sean turistas sexuales y hombres que sean turistas de romance?

Carr (2016) también considera a Hamid-Turksoy, Van Zoonen y Kuipers (2014) y Frohlick (2013) para decir que es una realidad en la que hasta hace relativamente poco tiempo las investigaciones en turismo sexual han estado enfocadas únicamente en hombres heterosexuales, ignorando la participación de las mujeres como consumidoras. A diferencia de Vargas y Alcalá (2015, p. 886), quien considera que "el principal factor de la prostitución masculina no ha sido el turismo, pero sí ha favorecido su crecimiento en los destinos turísticos". En este sentido, Córdova Plaza (2010) en Córdova Plaza (2015, p. 850) considera que a los jóvenes sin empleo y sin una carrera o que tienen un empleo de medio tiempo les resulta difícil encontrar un trabajo bien remunerado, por lo que recurren a la actividad sexual porque relativamente es fácil y se generan grandes ingresos por día. La prostitución masculina requiere de cierta discreción, a comparación de la femenina; la búsqueda de clientes puede ser más difícil en los primeros porque su principal objetivo es pasar desapercibidos ante la dinámica del espacio determinado. Córdova Plaza (2015, p. 850) recuperando a Khan (1990) establece que personas de bajos recursos que viven en diversas situaciones desfavorables son las que realizan la venta de sus cuerpos, ya que consideran que es su única salida para sobrevivir y poder tener una vida digna. Estas dos perspectivas implican consecuencias sociales e impactantes, en un primer sentido en la construcción y formación de la persona en lo individual y en segundo término en las relaciones sociales y familiares que establezcan a su alrededor.

Una de las principales consecuencias es que la sociedad en general vea como una perversión a los que están involucrados ya sea como trabajador, consumidor o asociado, lo que aumenta considerablemente los debates académicos al respecto, como lo mencionan Want (2002) y Ryan y Kinder (1996) citados por Carr (2016). Otro elemento muy importante y bastante popular dentro de las investigaciones sobre turismo sexual es la participación del turismo gay que se ha considerado como un buen mercado, en palabras de Hughes (2008) vistos como clientes favorables para los prestadores de servicios, incluso más que un mercado heterosexual, pues según el autor son personas con buen nivel socioeconómico y altos niveles de consumo, lo que los convierte en un mercado perfecto para ser atraído. En Estados Unidos y el Reino Unido es un nicho de mercado que representa un crecimiento considerable (Clift \& Forrest, 1999; Holcomb \& Luongo, 1996, citados en Hughes, 2008).

En México, Puerto Vallarta es importante a nivel internacional no solamente por la playa que tiene y la vida nocturna; sino por que ocupa el segundo lugar mundial en ser frecuentado por turistas homosexuales estadounidenses. Esto quiere decir que cuenta con los servicios que necesita el mercado gay (Hughes, Monterrubio, \& Miller, 2010; Torres, 2006; Vidal Aldana, 2007) citados en Mendoza (2015, p. 889).

\section{Turismo sexual: Rompiendo paradigmas}

Como lo establece Córdova Plaza (2015, p. 863), el turismo sexual no es un concepto aislado, posee una dinámica tan compleja y particular que ha provocado cambios en el ambiente hotelero y por supuesto en todo el fenómeno turístico y las industrias que lo rodean, impacta positiva y negativamente al destino, incluso moralmente, es una actividad que se percibe como riesgosa pero también de oportunidad (Chong, 2014). El concepto de turismo sexual como actividad económica no está aislada, en este sentido Mendoza (2005, p. 905) con base en los resultados presentados en su investigación, reafirma que en ciertos destinos, las actividades sexuales no son tan mal vistas y existen personas de lugares colindantes que se encuentran ofreciendo servicios sexuales en el destino. ¿Será posible que el turismo sexual rompa paradigmas en la manera de 


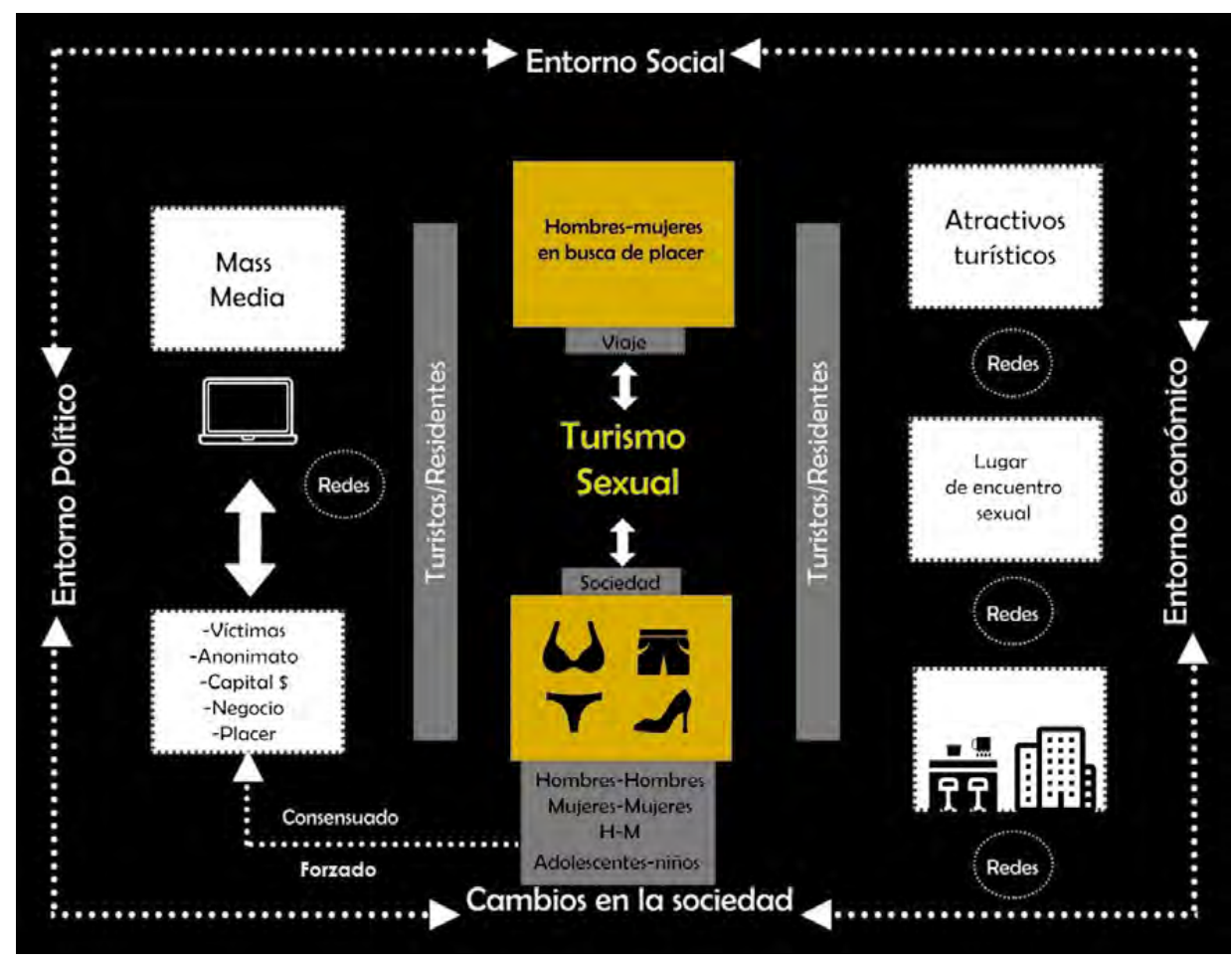

Figura 1. Se estudian el turismo sexual y sus implicaciones en varios entornos. Elaboración propia.

gestionar el tiempo libre, rompiendo formalidades sociales y morales al proveer experiencias "únicas" para los turistas?

La sociedad ha creado mitos y creencias a lo largo de la historia (Barthes, 1972, citado en Gezinski, Karandikar, Levitt, \& Ghaffarian, 2016) y en este sentido podría haber creado estándares de comportamiento humano con respecto al uso del tiempo libre que impacte en algo tan particularmente humano como el sexo. La creciente popularidad del turismo sexual ha provocado en los ofertantes de este servicio la capacidad de ofrecerlo bajo discreción como una condición de calidad; en este sentido, destinos con este perfil de turismo promueven sus servicios al visitante asegurando discreción y seguridad. Comparativamente Vargas y Alcalá $(2015$, p. 869) recuperando a varios autores (Carrascal \& Pérez, 1998; Escudero, 1997; INEGI \& Secretaría de Turismo, 1999; Ramírez, 1986; Ruiz, 1992; Valenzuela, 2008) mencionan que la industria hotelera fue creciendo, se crearon restaurantes, antros, clubes, lo que fue generando mayores puntos de encuentro para los prestadores de servicios sexuales; por tanto, se fueron diversificando los sitios que posibilitan la existencia de este tipo de turismo y de demanda turística.
Un dato importante que Chong (2014) propone es el impacto que puede llegar a tener el turismo sexual en un destino al influenciar la toma de decisiones por parte del turista, como por ejemplo, la elección de un hotel. Esto implica que la práctica estudiada podría modificar los estándares y características deseados por el viajante, y en este sentido la industria hotelera tendría que diversificar o adaptar su oferta para poder atender demandas específicas. Ciertamente el placer obtenido por medio de la práctica del turismo sexual es un tipo de placer que se encuentra catalogado en la taxonomía de los productos turísticos como una clase de producto específico para una familia de productos en turismo (McKercher, 2016).

Golding (2003) citado por Hughes (2008) ve a las comunidades lésbico-gay como un mercado aún poco explotado siendo que tienen una mayor tendencia a viajar y gastar más dinero en actividades recreativas que las comunidades heterosexuales. Esto significa que los destinos turísticos podrían ver hacia otro tipo de mercado con la finalidad de sobrevivir en las dinámicas turísticas, aunque caigan en prácticas que socialmente se consideran incorrectas, poco seguras, poco morales o simplemente son socialmente castigadas. 


\section{IIVESTIGAGIÓn Y CUERCIA DE LA UחIVERSIDAD AUTÓNOMA DE RGUASCHLIETTES}

CONCLUSIONES

Como acertadamente menciona Ryan (2009), corresponde a los investigadores académicos esclarecer los aspectos aún ocultos sobre el turismo sexual y las actividades sexuales relacionadas con el turismo (citado por Carr, 2016). Trabajar en la legislación de este tipo de turismo fomentaría una mejor gestión de la actividad, reconociendo su existencia en la dinámica turística de los destinos. Es una práctica que ha contaminado la esfera social, cuando se trata de negociar con el cuerpo de otros, han sido muchos los afectados por esta práctica, queda mucho por esclarecer y definir las relaciones entre el desplazamiento hacia un destino en busca de actividades o servicios sexuales y la prestación de estos al viajante.

Viajar otorga a los turistas un sentido de anonimato y libertad únicos que los anima a realizar conductas y actividades que normalmente no realizarían. El concepto estudiado puede llegar a convertirse en el atractivo mismo de un sitio. Para algunas personas, el turismo sexual consensuado, sea por el intercambio de un capital o no, representa una forma de liberación de deseos y frustraciones que no se permiten expresar en su vida cotidiana. El turismo sexual no debe ser limitativo hacia el género masculino.

Existe una evidente carencia de investigaciones sobre este tema donde se considere a las mujeres como usuarias y demandantes de los servicios sexuales, comúnmente el género femenino se encuentra fuertemente ligado al llamado turismo de romance, o bien, conformado en una plantilla como prestadoras de servicios sexuales. Es posible inferir que los visitantes lésbico-gay suelen ser los principales y más abiertos participantes de la dinámica turística sexual en muchos destinos, por este motivo deben tratarse como un segmento de mercado que diversifica a la realidad turística, sin limitarlo ni etiquetarlo socialmente. No se debe olvidar que este tipo de turismo existe, pues corresponde a una necesidad específica y a partir de aquí pueden surgir productos "turísticos" en los destinos que satisfacen este tipo de práctica.

\section{REFERENCIAS}

- Arroyo, L., \& Amador, K. (2015). turismo y trabajo sexual masculino en Cancún-México. Estudios y Perspectivas en Turismo, 24(3), 982-992. Recuperado de http://www. estudiosenturismo.com.ar/PDF/V24/N04/v24n4a12.pdf

- Bringas, N. L., \& Gaxiola, R. (2015). Códigos encubiertos. Prostitución de varones y turismo sexual en Tijuana, México. Estudios y Perspectivas en Turismo, 24(4), 825-847. Recuperado de https://dialnet.unirioja.es/servlet/articulo? codigo=5276662

- Cáceres, C. F., Nureña, C. R., \& Gomero, A. (2015). La oferta de trabajo sexual masculino en el Perú: Su interacción con las demandas del turismo internacional. Estudios y Perspectivas en Turismo, 24(4), 924-942. Recuperado de http://www.scielo.org.ar/scielo.php?script=sci_arttext\&pid $=$ S1851-17322015000400009

- Carr, N. (2016). Sex in tourism : Reflections and potential future research directions. Tourism Recreation Research, 41 (2), 188198. Recuperado de http://doi.org/10.1080/02508281.2016.11 68566

- Chong, K. L. (2014). The impact of sex tourism on Hotel Selection. International Journal of Tourism Sciences, 14(3), 106115. Recuperado de http://doi.org/10.1080/15980634.2014.11 434702
- Córdova Plaza, R. (2015). Universos paralelos. Turismo sexual comercial entre hombres en el Puerto de Veracruz, México. Estudios y Perspectivas en Turismo, 24 (4), 848-866. Recuperado de http://www.estudiosenturismo.com.ar/PDF/V24/N04/ v24n4a05.pdf

- DDD. (25 de noviembre de 2017). Toluca: Desarticulan red de explotación sexual de mujeres latinoamericanas. Regeneración. Recuperado de https://regeneracion.mx/ toluca-red-explotacion-sexual/

- França, I. L. (s. f.). Diferencias y desigualdades en los desplazamientos. Turistas e inmigrantes gay brasileños entre São Paulo (Brasil) y Barcelona (España). Estudios y Perspectivas en Turismo, 24(4), 963-981. Recuperado de http://www. estudiosenturismo.com.ar/PDF/V24/N04/v24n4al 1.pdf

- Gezinski, L. B., Karandikar, S., Levitt, A., \& Ghaffarian, R. (2016). "Total girlfriend experience": Examining marketplace mythologies on sex tourism websites. Culture, Health \& Sexuality, 18(7), 785-798. Recuperado de http://doi.org/10.108 0/13691058.2015.1124457

- Huang, S., Van der Veen, R., \& Zhang, G. (2014). New Era of China Tourism Research. Journal of China Tourism Research, 10(4), 379-387. doi: 10.1080/19388160.2014.952909 
- Hughes, H. L. (2008). A gay tourism market. Journal of Quality Assurance in Hospitality \& Tourism, 5(2/4), 57-74. Recuperado de http://doi.org/10.1300/J162v05n02

- Jeffreys, S. (2003). Sex tourism: Do women do it too? Leisure Studies, 22(3), 223-238. Recuperado de http://doi. org/10.1080/026143603200075452

- Lagunas, D. (2010). El poder del dinero y el poder del sexo. Antropología del turismo sexual. Perfiles Latinoamericanos, 36, 71-98. Recuperado de Www.redalyc.org/ pdf/115/11515386003.pdf

- Lanzarini, R. (2015). Homoerotismo durante los viajes: El placer sexual entre hombres en espacios anónimos en Brasil y Portugal. Estudios y Perspectivas en Turismo, 24(4), 943-962. Recuperado de http://www.estudiosenturismo.com.ar/PDF/ V24/N04/v24n4a10.pdf

- McKercher, B. (2016). Towards a taxonomy of tourism products. Tourism Management, 54, 196-208.

- Mendoza, C. (2015). Entre el negocio y el placer. Turismo sexual masculino en Puerto Vallarta, México. Estudios y Perspectivas en Turismo, 24(4), 889-907. Recuperado de http://www. estudiosenturismo.com.ar/PDF/V24/N04/v24n4a07.pdf

- Monterrubio, J. C. (2015). Construcciones socioculturales de la playa nudista. Entre lo liminar y el homoerotismo. Estudios y Perspectivas en Turismo, 24 (4), 908-923. Recuperado de http:// www.estudiosenturismo.com.ar/PDF/V24/N04/v24n4a08.pdf

- Oppermann, M. (1999). Sextourism. Annals of Tourism Research, 26(2), 251-266. Recuperado de http://doi.org/10.1016/S01607383(98)00081-4
- Quintero Venegas, G. J., López López, A., \& Gallegos Jiménez, O. (2015). Cuerpos de hombres en venta en ámbitos turísticos de la Ciudad de México. Una perspectiva espacial. Estudios y Perspectivas en Turismo, 24(4), 809-824. Recuperado de http://www.scielo.org.ar/scielo.php?script=sci_arttext\&pid $=$ S1851-17322015000400003

- Redacción. (26 de noviembre de 2017). Extranjeras explotadas cubrían cuota de cinco clientes al día. Excélsior. Recuperado de https://www.excelsior.com.mx/ comunidad/2017/11/26/1203586

- Sánchez Mendoza, V. G., \& Santamaría Gómez, A. (2015). Sexo, sol y playa. El inconsciente del imaginario turístico en Mazatlán-México. Estudios y Perspectivas en Turismo, 24(4), 993-1013. Recuperado de https://dialnet.unirioja.es/servlet/ articulo? codigo $=5276654$

- Van Broeck, A. M., \& López López, A. (2015). Turismo y sexo. Una reflexión teórica desde el homoerotismo y el espacio. Estudios y Perspectivas en Turismo, 24 (4), 787-808. Recuperado de http://www.scielo.org.ar/scielo.php?script=sci_arttext\&pid $=$ S1851-17322015000400002

- Vargas, S. Y., Alcalá, B. (2015). Aspectos territoriales de la prostitución masculina vinculada al turismo sexual en Acapulco. Estudios y Perspectivas en Turismo, 24(4), 867-888. Recuperado de http://www.estudiosenturismo.com.ar/PDF/ v24/N04/v24n4a06.pdf

- Xin, S., Tribe, J., \& Chambers, D. (2013). Conceptual research in tourism. Annals of Tourism Research, 41, 66-88. Recuperado de http://doi.org/10.1016/j.annals.2012.12.003 